\title{
Fifth finger camptodactyly maps to chromosome 3q11.2-q13.12 in a large German kindred
}

\author{
Sajid Malik*,1,2 ${ }^{\star}$ Jörg Schott ${ }^{1}$, Julia Schiller ${ }^{1}$, Anna Junge ${ }^{1}$, Erika Baum ${ }^{3}$ and \\ Manuela C Koch ${ }^{1}$
}

${ }^{1}$ Zentrum für Humangenetik, Philipps-Universität Marburg, Bahnhofstr. 7, Marburg, Germany; ${ }^{2}$ Department of Animal Sciences, Quaid-I-Azam University Islamabad, Islamabad, Pakistan; ${ }^{3}$ Abteilung für Allgemeinmedizin, Präventive und Rehabilitative Medizin, Philipps-Universität Marburg, Robert-Koch-Str. 5, Marburg, Germany

Camptodactyly (MIM 114200) is a digit deformity characterised by permanent flexion contracture of fifth fingers at the proximal interphalangeal (PIP) joints. The sporadic cases are common but a familial occurrence is not much appreciated. In an attempt to identify the genetic basis of camptodactyly, we have analysed a large German family with camptodactyly segregating in an autosomal dominant fashion. The affected family members exhibited clinical features of fifth finger camptodactyly and knuckle pads on the crooked fifth finger and on fingers 2-3. Typically, women were more severely affected than men.

Microsatellite analyses of five candidate loci known to be associated with camptodactyly-like phenotypes did not show co-segregation with the phenotype in our family. A genome-wide linkage scan using a total of $\mathbf{4 1 4}$ microsatellite markers gave significant evidence of linkage between the familial phenotype and chromosomal locus 3q11.2-q13.12 (maximum two-point LOD score 3.04). The key recombination events showed that the phenotype localises between markers D3S2465 and D3S3044, spanning an interval of $\sim 15 \mathrm{cM}$. This study reports the first genetic locus linked to isolated autosomal dominant fifth finger camptodactyly with knuckle pads and proves the hypothesis that camptodactyly is distinct from camptodactyly-associated phenotypes including Dupuytren contracture. Additional studies of other families will be necessary to determine the existence of genetic homogeneity or heterogeneity of the anomaly and to narrow down the genetic interval to identify the responsible gene. Since genetic heterogeneity for isolated camptodactyly is likely, we propose to designate the 3q11.2-q13.12 locus as CAMPD1 (ie, camptodactyly 1). European Journal of Human Genetics (2008) 16, 265-269; doi:10.1038/sj.ejhg.5201957; published online 14 November 2007

Keywords: camptodactyly; knuckle pads; linkage 3q11.2-q13.12; locus CAMPD1

Introduction

Camptodactyly (MIM 114200) is defined as a permanent flexion contracture of one or both fifth fingers at the proximal interphalangeal (PIP) joints. Additional fingers

*Correspondence: Dr S Malik, Zentrum für Humangenetik, PhilippsUniversität Marburg, Bahnhofstr. 7, Marburg 35037, Germany.

Tel: + 496421286 6704; Fax: + 496421286 4082;

E-mail: malik@staff.uni-marburg.de

Received 15 August 2007; revised 12 October 2007; accepted 17 October 2007; published online 14 November 2007 might be affected, but the little finger is always involved. If all fingers of both hands show contractures some authors call the anomaly streblodactyly. ${ }^{1-4}$ The relevant references suggest that camptodactyly and streblodactyly are used interchangeably, while camptodactyly is the more common term..$^{5-7}$ A third term, streblomicrodactyly seems to be reserved for the isolated crooked little finger, but is rarely used. ${ }^{8}$

The phenotype of camptodactyly can be a part of defined syndromes (Table 1). Camptodactyly must be distinguished from Dupuytren contracture (MIM 126900), 
Table 1 Chromosomal localisation of hereditary disorders with camptodactyly

\begin{tabular}{|c|c|c|c|c|}
\hline OMIM & Syndrome & Description & Inheritance & Locus (gene) \\
\hline 193700 & $\begin{array}{l}\text { Arthrogryposis distal, } \\
\text { type } 2 \mathrm{~A} \text {, Freeman-Sheldon }\end{array}$ & Craniotarsal dysplasia, whistling face & $A D$ & $17 p 13.1$ \\
\hline 208250 & CACP Jacobs syndrome & $\begin{array}{l}\text { Camptodactyly-arthropathy-coxa vara- } \\
\text { pericarditis }\end{array}$ & AR & $1 \mathrm{q} 24-\mathrm{q} 25(P R G 4)$ \\
\hline
\end{tabular}

a fibroproliferative disorder of the palmar fascia with severe disfigurement of hands and fingers. Knuckle pads (MIM 149100), subcutaneous nodules over the dorsal PIP joints, are commonly associated with Dupuytren contracture, but they also might be observed in combination with isolated camptodactyly. ${ }^{4,5}$

Isolated camptodactyly is not a rare condition, but is frequently overlooked especially if it is restricted to the fifth finger and does not interfere with the function of the hand. Usually, the condition appears to be sporadic in a family, but clinical examination of relatives reveals that it is an autosomal dominant condition with variable penetrance and expressivity. In contrast to Dupuytren contracture, men are more mildly affected than women. ${ }^{3,4}$ Finger deformities, especially camptodactyly were featured in Renaissance art. Examples are the brush drawing Betende Hände of the German artist Albrecht Dürer and the paintings of the Dutch artist Dirk Bouts. ${ }^{9}$

Here, we report a large German kindred with typical features of isolated fifth finger camptodactyly, knuckle pads and a preponderance in women. The condition is segregating as an autosomal dominant trait and was localised to chromosome 3q11.2-q13.12 by linkage analysis. This locus is not shared by any other camptodactylylike syndrome (Table 1).

\section{Family ascertainment and clinical characteristics}

The family originates from Hessen, Germany. Thirteen members ( 8 female, 5 male) in four successive generations are affected by isolated fifth finger camptodactyly and knuckle pads (Figure 1). Nineteen individuals, including nine unaffected, were physically examined. The status of each individual was ascertained in a home visit. Physical examination followed a standardised protocol including a detailed medical history. Associated defects such as facial anomalies, other joint contractures or skeletal defects were not observed in any family member. Blood samples from 17 individuals (10 affected and 7 unaffected) were obtained for molecular analysis. All family members provided written informed consent according to the approved study protocol of the ethics committee of the University of Marburg.
The phenotype is characterised by bilateral, low-set fifth fingers with contractures at the PIP joint without involvement of other fingers (Figure 2, Table 2). The flexion at the PIP joint is accompanied by hyperextension at the metacarpophalangeal and the terminal interphalangeal joint. Individuals stated that after juvenile age, the contracture did not progress further. In addition, almost all of the affected individuals have knuckle pads upon the PIP joints, most prominent on the crooked fifth finger and on fingers $2-3$. The pads are less marked on the ring finger and are never present on the thumb. These pads were not observed in non-affected family members. In female members, the flexion anomaly started in early childhood deteriorating slowly with age. Affected male members generally show a milder phenotype. Individuals IV-3, IV-7 and IV-9 have prominent knuckle pads on fingers $2-3$ and mild ones on the ring finger, thus revealing the clinical status. At the time of examination, the female individual V-4 (age 15 years) had not yet developed knuckle pads. Male individuals V-5 (8 years) and V-6 (4 years) were designated clinically uncertain since they did not show either camptodactyly or knuckle pads. Zygodactyly, 2/3 toe soft-tissue webbing, was observed in three family members (III-2, IV-2 and IV-5) and was regarded as coincidental.

\section{Genotyping and linkage analysis}

Genomic DNA from blood was extracted according to standard methods. To map the phenotype in an initial approach, microsatellite markers flanking candidate loci for camptodactyly-like phenotypes on chromosomes $1 \mathrm{q}$, $4 p, 16 q, 17 p$ and $17 q$ were selected (Table 1 ). In the second step, a genome-wide scan was conducted by employing a panel of 400 highly polymorphic autosomal microsatellite markers with an average inter-marker distance of $8.7 \mathrm{cM}$. Genotyping methods, including PCR conditions, multiplexing, allele separation and scoring, were essentially the same as described earlier. ${ }^{10}$

For fine mapping, 14 additional markers were used, nine being on chromosome 3q (Table 3). For linkage analysis, file formatting was done with Mega2 version 3.0. ${ }^{11}$ Twopoint LOD score calculations were performed by using the MLINK program FASTLINK version 4.1. ${ }^{12,13}$ SIMWALK2 


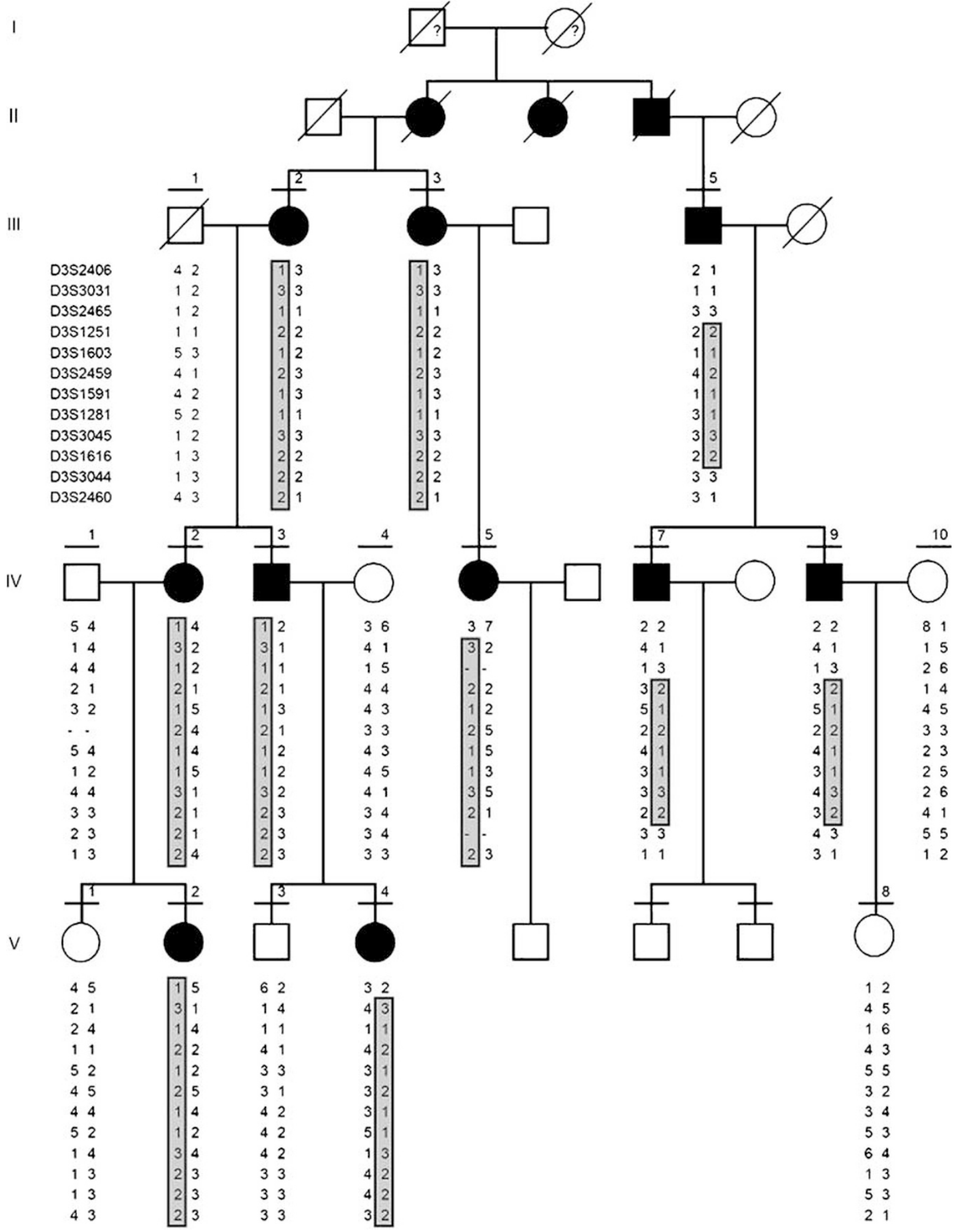

Figure 1 Pedigree of the German family with autosomal dominant camptodactyly. The subjects clinically examined are depicted with a horizontal bar above the symbols. Genotypes of 12 microsatellite markers from chromosome $3 q$ are provided, depicting a common haplotype segregating in the affected subjects. 

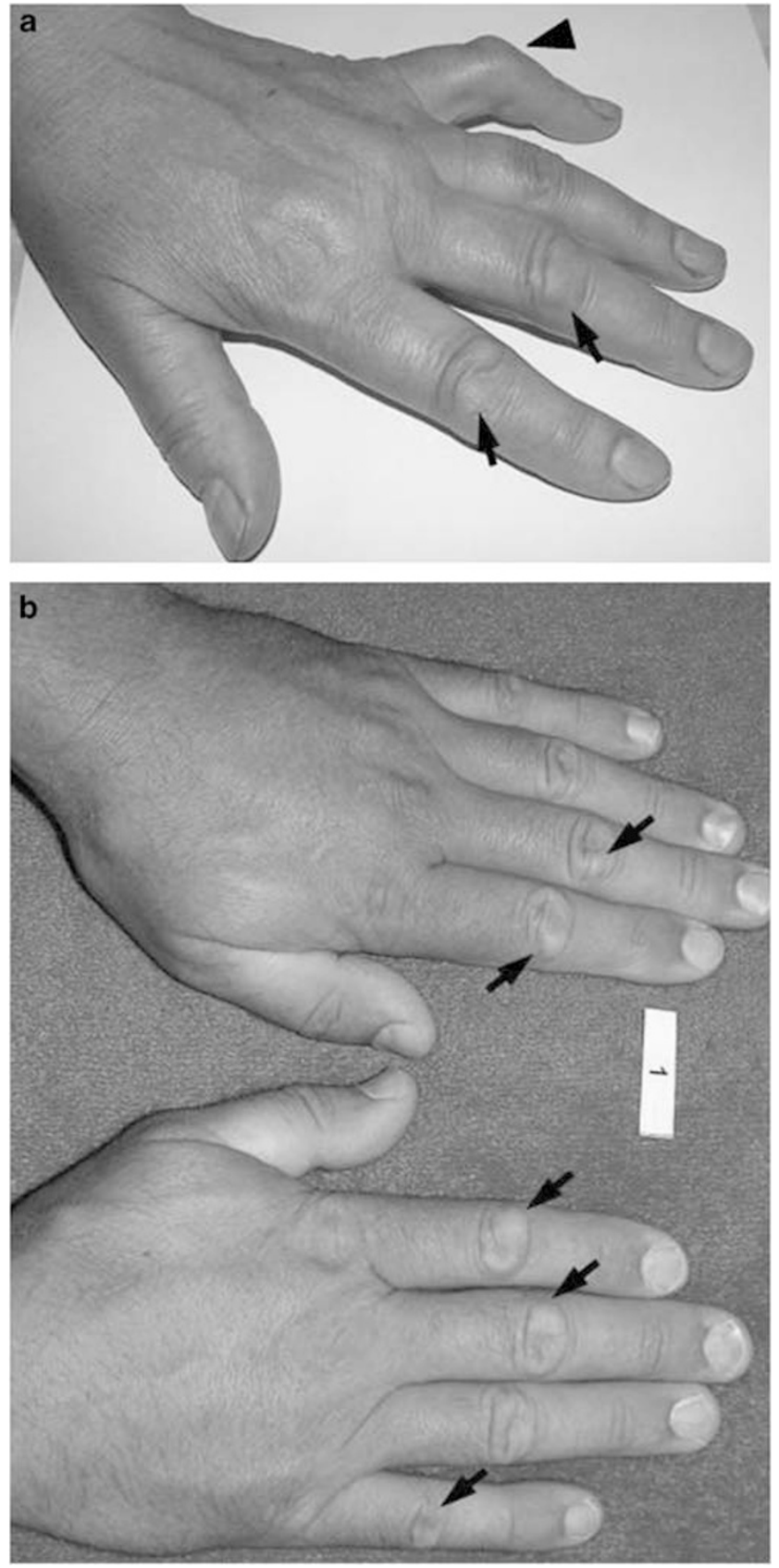

Figure 2 Camptodactyly phenotype observed in the German family. Fifth finger contracture marked with an arrow head, and knuckle pads indicated with filled arrows. (a) Subject III-2. (b) Subject IV-9.

version 2.83 was used to calculate multipoint scores and to generate haplotypes with the highest likelihood. ${ }^{14}$ Analyses were repeated with Superlink. ${ }^{15}$ The phenotype was coded as an autosomal dominant trait with a penetrance of 0.999 and a disease frequency of 0.0001 . Marker allele frequencies were assumed to be equal. To avoid a bias of linkage estimate, no allele was allowed to be $<10 \%$.
Table 2 Camptodactyly phenotype in the German family

\begin{tabular}{lccc}
\hline Individual & Gender & $\begin{array}{c}\text { Phenotype } \\
\text { Fifth finger } \\
\text { camptodactyly }\end{array}$ & $\begin{array}{c}\text { Knuckle } \\
\text { pads }\end{array}$ \\
\hline III-2 & F & ++ & + \\
III-3 & F & + & + \\
III-5 & M & + & ++ \\
IV-2 & F & + & + \\
IV-3 & M & - & + \\
IV-5 & F & + & + \\
IV-7 & M & - & ++ \\
IV-9 & F & - & ++ \\
V-2 & F & ++ & + \\
V-4 & F & + & - \\
\hline
\end{tabular}

+ mild; ++ prominent; - absent.

\section{Results and discussion}

Here, we describe an autosomal dominantly inherited phenotype of isolated fifth finger camptodactyly with knuckle pads in a four-generation family. As described in previous reports, ${ }^{4-7}$ fifth finger camptodactyly in this family was more prominent in women than in men, and indeed the family came to our attention through the female individual III-2. In female members, camptodactyly began to develop in early childhood and the full contracture was manifested before adulthood. In all individuals, the contracture was confined to the fifth finger. Knuckle pads upon PIP joints were an integral part of the phenotype. These knuckle pads were associated with the crooked finger and were also evident on fingers 2 and 3, and to a lesser degree on the ring finger but never on the thumb. In three male members, the knuckle pads were obvious, and there was suspicion of a mild contracture and hyperextension of the distal joint of the fifth finger. The family members were not aware that these pads were associated with the camptodactyly. Involvement of the palmar fascia was not observed in any of the individuals, and therefore, Dupuytren contracture was definitely ruled out.

Before starting on a genome-wide microsatellite scan to localise the condition in the human genome, five candidate loci of disorders with camptodactyly were excluded for co-segregation with the phenotype in our family (Table 1). A genome-wide search initially revealed five loci having an LOD score $>1.0$, including a locus on chromosome 3q. The fine mapping of the $3 \mathrm{q}$ region indeed revealed a large chromosomal interval segregating in all the affected family members. The highest two-point LOD score was obtained with marker D3S1251 (3.04 at $\theta=0.00$; Table 3). The multipoint analysis yielded a maximum LOD score of 3.64 at $124 \mathrm{cM}$ (data not shown). Re-analyses of the genotype data with Superlink ${ }^{15}$ produced the same results. Haplotype analysis depicted key recombination events between markers D3S2456 and D3S1251 in individuals III-5, IV-7 and IV-9, defining the upper limit of the locus, 
Table 3 Two-point LOD scores for camptodactyly and microsatellite markers on chromosome 3q11.2-q13.12

\begin{tabular}{|c|c|c|c|c|c|c|c|c|c|}
\hline \multirow[b]{2}{*}{ Marker } & \multirow[b]{2}{*}{ UCSC } & \multirow[b]{2}{*}{$c M$} & \multicolumn{7}{|c|}{ Recombination fraction $(\theta)$} \\
\hline & & & 0.00 & 0.01 & 0.05 & 0.10 & 0.20 & 0.30 & 0.40 \\
\hline D3S2406 & 73341066 & 102.64 & -8.75 & -3.28 & -1.31 & -0.57 & -0.02 & 0.13 & 0.10 \\
\hline D3S3031 & 80406618 & 109.22 & -1.42 & 0.32 & 0.88 & 1.00 & 0.93 & 0.70 & 0.39 \\
\hline D3S2456 & 84748919 & 111.89 & -2.02 & -0.25 & 0.33 & 0.50 & 0.53 & 0.41 & 0.23 \\
\hline D3S1251 & 95759698 & 114.02 & 2.03 & 1.99 & 1.84 & 1.64 & 1.24 & 0.83 & 0.42 \\
\hline D3S1603 & 99942318 & 115.09 & 2.74 & 2.69 & 2.49 & 2.22 & 1.67 & 1.11 & 0.55 \\
\hline D3S2459 & 103657974 & 119.09 & 2.14 & 2.11 & 1.98 & 1.80 & 1.37 & 0.91 & 0.44 \\
\hline D3S1591 & 106807838 & 121.67 & 3.04 & 2.99 & 2.76 & 2.48 & 1.88 & 1.25 & 0.61 \\
\hline D3S1281 & 107459037 & 124.16 & 2.41 & 2.36 & 2.15 & 1.90 & 1.37 & 0.85 & 0.39 \\
\hline D3S3045 & 108472547 & 124.16 & 2.13 & 2.09 & 1.93 & 1.72 & 1.29 & 0.86 & 0.43 \\
\hline D3S1616 & 109086951 & 124.16 & 2.03 & 1.99 & 1.84 & 1.64 & 1.24 & 0.83 & 0.42 \\
\hline D3S3044 & 112445090 & 126.83 & -5.12 & -1.66 & -0.39 & 0.06 & 0.34 & 0.34 & 0.22 \\
\hline D3S2460 & 118885028 & 134.64 & -4.07 & -0.70 & 0.48 & 0.82 & 0.87 & 0.65 & 0.34 \\
\hline
\end{tabular}

while the lower boundary was defined by the recombination between markers D3S1616 and D3S3044, also observed in the right loop of the pedigree (individuals III-5, IV-7 and IV-9). Therefore, the associated locus in this family flanks markers D3S2406 and D3S3044, spanning an interval of $14.94 \mathrm{cM}(27.69 \mathrm{Mb})$.

Our molecular data prove the previous hypothesis by Welch and Temtamy ${ }^{4}$ that fifth finger camptodactyly with knuckle pads is genetically distinct from streblodactylyand camptodactyly-associated phenotypes including Dupuytren contracture (Table 1). Additional studies of other families will be necessary to determine the existence of genetic homogeneity or heterogeneity of the anomaly and to narrow down the genetic interval to identify the responsible gene. Since genetic heterogeneity for isolated camptodactyly is likely, we propose to designate the 3q11.2-q13.12 locus as CAMPD1 (ie, camptodactyly 1).

\section{Acknowledgements}

We thank the family for their participation in the study and Gerda Panzner for technical support. The study was supported by the Deutsche Forschungsgemeinschaft (Graduiertenkolleg GRK 767).

\section{References}

1 Parish JG, Horn DB, Thompson M: Familial streblodactly with amino-aciduria. BMJ 1963; 2: 1247-1250.
2 Donofrio P, Ayala F: Familial streblodactyly. Acta Derm Venereol 1983; 63: 361-363.

3 Spear GS: The inheritance of flexed fingers. $J$ Hered 1946; 37: 189-192.

4 Welch JP, Temtamy SA: Hereditary contractures of the fingers (camptodactyly). J Med Genet 1966; 3: 104-113.

5 Temtamy SA, McKusick VA: Contracture deformaties as isolated anomalies. In Temtamy SA, McKusick VA (eds): The Genetics of Hand Malformations. New York: Alan R Liss, 1978, pp 441-446.

6 Schallreuter KU, Reimlinger S: [Camptodactyly. The syndrome of flexion contracture of the fingers]. Hautarzt 1993; 44: 157-159.

7 Brites MM, Moreno A, Salgado M et al: Familial camptodactyly. Eur J Dermatol 1998; 8: 355-356.

8 Hefner RA: Crooked little finger (minor streblomicrodactyly). J Hered 1941; 32: 37-38.

9 Hijmans W, Dequeker J: Camptodactyly in a painting by Dirk Bouts (c. 1410-1475). J R Soc Med 2004; 97: 549-551.

10 Malik S, Percin FE, Ahmad W et al: Autosomal recessive mesoaxial synostotic syndactyly with phalangeal reduction maps to chromosome 17p13.3. Am J Med Genet A 2005; 134: 404-408.

11 Mukhopadhyay N, Almasy L, Schroeder M et al: Mega2. A datahandling program for facilitating genetic linkage and association analyses. Am J Hum Genet 1999; 65: A436.

12 Cottingham Jr RW, Idury RM, Schäffer AA: Faster sequential genetic linkage computations. Am J Hum Genet 1993; 53: 252-263.

13 Schaffer AA, Gupta SK, Shriram K et al: Avoiding recomputation in linkage analysis. Hum Hered 1994; 44: 225-237.

14 Sobel E, Lange K: Descent graphs in pedigree analysis: applications to haplotyping, location scores, and marker-sharing statistics. Am J Hum Genet 1996; 58: 1323-1337.

15 Fishelson M, Geiger D: Exact genetic linkage computations for general pedigrees. Bioinformatics 2002; 18 (Suppl 1): S189-S198. 